\title{
Traumatic brain injury-induced sleep disorders
}

This article was published in the following Dove Press journal:

Neuropsychiatric Disease and Treatment

I5 February 2016

Number of times this article has been viewed

\section{Mari Viola-Saltzman \\ Camelia Musleh}

Department of Neurology, NorthShore University HealthSystem, Evanston, IL, USA
Correspondence: Mari Viola-Saltzman Department of Neurology, NorthShore University HealthSystem, 2180 Pfingsten Road, Glenview, IL 60026, USA

Tel + I 8476638200

Fax + I 8476575767

Email mviolasaltzman@northshore.org

\begin{abstract}
Sleep disturbances are frequently identified following traumatic brain injury, affecting $30 \%-70 \%$ of persons, and often occur after mild head injury. Insomnia, fatigue, and sleepiness are the most frequent sleep complaints after traumatic brain injury. Sleep apnea, narcolepsy, periodic limb movement disorder, and parasomnias may also occur after a head injury. In addition, depression, anxiety, and pain are common brain injury comorbidities with significant influence on sleep quality. Two types of traumatic brain injury that may negatively impact sleep are acceleration/deceleration injuries causing generalized brain damage and contact injuries causing focal brain damage. Polysomnography, multiple sleep latency testing, and/or actigraphy may be utilized to diagnose sleep disorders after a head injury. Depending on the disorder, treatment may include the use of medications, positive airway pressure, and/or behavioral modifications. Unfortunately, the treatment of sleep disorders associated with traumatic brain injury may not improve neuropsychological function or sleepiness.
\end{abstract}

Keywords: traumatic brain injury, insomnia, hypersomnia, sleep apnea, periodic limb movement disorder, fatigue

\section{Introduction}

Sleep disturbances are frequently identified following traumatic brain injury, affecting $30 \%-70 \%$ of persons, and often occur after mild head injury. ${ }^{1-3}$ Traumatic brain injury is a significant cause of disability and death worldwide. The Glasgow Coma Scale scores traumatic brain injury as mild, moderate, or severe (mild $=13-15$, moderate $=9-12$, severe $=\leq 8$ out of 15$).{ }^{4}$ Brain injury can result in significant sensory, motor, cognitive, and emotional impairments. Even mild traumatic brain injury can be associated with dizziness, headache, nausea/vomiting, impaired balance and coordination, vision changes, tinnitus, mood and memory changes, difficulty with memory and attention, fatigue, and/or sleep disturbances. The relationship between head injuries and altered consciousness and cognitive changes has been well described, but the association between head trauma and sleep disturbance has not been extensively researched. ${ }^{5}$

\section{Epidemiology and risk factors}

Traumatic brain injuries are most commonly caused by falls $(28 \%)$, motor vehicle accidents (20\%), impact from an object (19\%), and assaults $(11 \%) .{ }^{6}$ These injuries often are related to athletic injuries, construction or industrial accidents, and domestic (and child) abuse. There is increasing awareness of traumatic brain injury in deployed military. Among the US military serving abroad, 11\%-23\% have suffered mild traumatic brain injury, often from improvised explosive device blasts. ${ }^{7}$

The exact prevalence of specific posttraumatic sleep disorders is not well defined. Baumann et al discovered that three out of four patients who were initially hospitalized for traumatic brain injury developed sleep-wake disturbances by 6 months postinjury. 
The majority of these patients had symptoms of hypersomnia or fatigue, while insomnia was present in only $5 \% .{ }^{8}$ However, other authors have identified a higher prevalence of insomnia after head injury. In a study that examined 60 patients with sleep-related complaints 3-24 months after traumatic brain injury (severity of traumatic brain injury was $40 \%$ mild, $20 \%$ moderate, and $40 \%$ severe), $25 \%$ of the participants complained of insomnia. ${ }^{9}$

Children also report sleep issues after suffering traumatic brain injury. One hundred and sixteen children hospitalized for mild traumatic brain injury were immediately evaluated, and $39 \%-67 \%$ of the population reported fatigue, trouble with sleep onset, drowsiness, and/or sleeping more or less than usual. At follow-up visit (2-3 weeks after injury), the sleep-related symptoms improved but persisted in $22 \%-38 \%{ }^{10}$ In a large study of 681 children who suffered from mild-to-severe traumatic brain injury, $14.7 \%$ reported sleep problems at 1-month follow-up. This percentage reduced to $10.7 \%$ at 4 -month follow-up and no patients reported sleep issues at 10-month follow-up. ${ }^{11}$ Finally, in another large study of 729 children with mild-to-severe traumatic brain injury compared to 197 patients with orthopedic injury, the traumatic brain injury group experienced a more prolonged duration and higher severity of sleep disturbances at 3,12 , and 24 months. ${ }^{12}$

Hou et al studied 98 posttraumatic brain injury patients to investigate the risk factors associated with the development of sleep disturbances. Risk factors for sleep disturbances after traumatic brain injury included severity of the head injury (as measured by the Glasgow Coma Scale), lower years of education, and the presence of residual symptoms (ie, headache and/or dizziness). Neither loss of consciousness or cause of injury was correlated with the development of sleep disturbances. The presence of residual headache, dizziness, anxiety, and/or depression was independently associated with the development of insomnia. The Glascow Coma Scale score was independently associated with hypersomnia. ${ }^{13}$

\section{Pathophysiology}

Traumatic brain injuries are characterized as primary or secondary brain injuries. A primary brain injury refers to the structural damage created during the time of impact from contact, acceleration-deceleration, and/or rotational forces. A secondary brain injury refers to the damage sustained from the subsequent cellular processes that occur from the primary injury (ie, hypoxia and/or raised intracranial pressure). ${ }^{14}$

The collision forces that act on the site of injury may lead to focal or diffuse injury. Focal injuries may include contact contusions, subdurals, epidurals, and/or intraparenchymal hemorrhages. Diffuse injuries are often caused by acceleration-deceleration forces that can cause shearing forces and widespread axonal injury in the brain. ${ }^{14}$ The location of injury within sleep-regulating brain regions leads to a specific sleep symptom. There is a paucity of clinicopathological correlative studies; however, those that do exist indicate several correlations. For instance, hypersomnia can result when areas involving the maintenance of wakefulness are injured. These regions include the rostral pons, caudal midbrain, and thalamus. ${ }^{15}$ In fact, closed head injury is known to lead to "shearing" forces along the direction of main fiber pathways causing microhemorrhages in these areas. ${ }^{16}$ Sleepiness and sleep attacks have also been linked to high cervical cord lesions, possibly by disruption of breathing during sleep. ${ }^{17}$ Interestingly, in a study that examined head trauma patients with hypersomnia, sleep-disordered breathing was found in all whiplash patients and thought to have occurred postinjury. ${ }^{18}$

In head injury, a coup injury exists at the brain's site of impact and a contrecoup injury involves the area of the brain opposite to the initial site of injury. A contrecoup injury usually occurs at the base of the skull. ${ }^{19,20}$ This type of injury occurs in areas of bony irregularities (especially the sphenoid ridges), with subsequent damage to the anterior temporal and inferior frontal regions, ${ }^{19,20}$ including the basal forebrain, which is involved in sleep initiation. Therefore, an injury in this area can lead to symptoms of insomnia. ${ }^{21}$ Sleep-wake disturbances after mild traumatic brain injury are associated with a longer tentorial length and flatter tentorial angle (the angle formed between the tentorium and a line through the foramen magnum) compared to patients with mild traumatic brain injury without sleep-wake disturbances. The length of time to recovery was directly correlated with tentorial length and indirectly correlated with tentorial angle. The authors postulated that in mild traumatic brain injury, direct impact involving the tentorium and pineal gland may result in the disruption of melatonin homeostasis and sleep-wake disturbances. ${ }^{22}$

Traumatic brain injury may compromise the nucleus suprachiasmatica and/or its output. This may cause disturbances in the circadian rhythm and, subsequently, a combination of hypersomnia and insomnia. A complete reversal of the circadian rhythmicity has been reported. ${ }^{23}$ In one case, a 20-year-old man suffered mild-to-moderate traumatic brain injury and penetrating eye injuries after an improvised explosive device blast. He was found to have a free-running type of circadian rhythm and was successfully treated with 
evening melatonin. ${ }^{24}$ Another study of 23 traumatic brain injury patients found lower levels of evening melatonin production compared to age- and sex-matched controls. ${ }^{25}$ Traumatic head injury has also been shown to be associated with alterations in the hypothalamic-pituitary-adrenal axis circadian rhythm. ${ }^{26}$

Low levels of cerebral spinal fluid hypocretin-1 are associated with narcolepsy with cataplexy but not with other types of central hypersomnias. ${ }^{27}$ For example, hypocretin-1 levels are usually normal in idiopathic hypersomnia. Low $(<110 \mathrm{pg} / \mathrm{mL})$ or intermediate $(110-200 \mathrm{pg} / \mathrm{mL})$ levels of hypocretin-1 have been found in a number of persons with neurologic lesions or diseases affecting the hypothalamus; some of these patients exhibit both sleepiness and cataplexy. ${ }^{28}$ Moderate-to-severe traumatic brain injury patients are usually found to have low or intermediate levels of hypocretin-1 in the acute phase of injury, but these levels tend to normalize (become $>200 \mathrm{pg} / \mathrm{mL}$ ) by 6 months after the insult. ${ }^{8}$

Rodrigues and Silva published a case of a patient who suffered from aggressive body movements during rapid eye movement sleep and periodic limb movements following a traumatic brain injury. Short sleep latencies were seen on multiple sleep latency test. The authors postulated that this patient's head injury was associated with alterations in the dopaminergic pathways and suggested the possibility of hypothalamic hypocretin involvement in its pathophysiology. ${ }^{29}$

\section{Clinical features and diagnosis}

Head trauma may result in significant sleep disturbance, more frequently in those suffering from mild head injury compared to those from more severe head injury. Mahmood et al examined 87 patients with mild-to-severe traumatic brain injuries and found that people with mild injury met the criteria for sleep disturbance more often than those with moderate or severe injury. One reason for this may be that patients with more severe forms of traumatic brain injury possibly reported less sleep disturbance because they were less aware of their sleep problems. Furthermore, patients with mild traumatic brain injuries were likely to complete the rehabilitation process quicker than those suffering from more severe traumatic brain injuries and thus face more imminent pressures to reintegrate into society. These psychosocial factors may lead to increased reporting of sleep disturbances in this population. Finally, organic differences in the neurological insults seen in mild and severe traumatic brain injuries may lead to different sleep disturbances..$^{30,31}$ Moreover, it is common for posttraumatic patients to complain of initial headaches that may lead to persistent and widespread pain or centralized pain that can have effects on sleep quality. ${ }^{32}$ Beta and gamma electroencephalogram (EEG) activity were found to be dominant in all sleep stages over the frontal, central, and occipital regions in posttraumatic patients with pain. ${ }^{33}$

\section{Insomnia}

One of the more common patterns following head injury involves trouble initiating and maintaining sleep, with or without subjective daytime sleepiness. In a study of 452 patients with traumatic brain injury, 50\% endorsed insomnia symptoms. ${ }^{34}$ In some patients, insomnia was a manifestation of a circadian rhythm sleep disorder, typically delayed sleep-wake phase disorder or irregular sleep-wake rhythm disorder. ${ }^{35}$ Among male military personnel, multiple brain injuries were found to lead to an increased risk for insomnia. ${ }^{36}$ Veterans with posttraumatic stress disorder and mild traumatic brain injury may be troubled by nightmares. ${ }^{37}$ Former US forces involved in Operation Enduring Freedom (Afghanistan) and Operation Iraqi Freedom (Iraq), who complained of insomnia associated with mild traumatic brain injury and posttraumatic stress disorder, were subjectively found to be sleepier compared to veterans with insomnia due to posttraumatic stress disorder alone. ${ }^{38}$ However, resolution of headaches and other neurologic deficits in veterans correlated with improvement of posttraumatic stress disorder symptoms and daytime sleepiness. ${ }^{39}$

\section{Hypersomnia}

Hypersomnia may develop after a head injury. If hypersomnia is present for at least 3 months, multiple sleep latency testing proves a mean sleep latency of 8 minutes or less, and there is no other obvious cause (ie, another sleep disorder, psychiatric disorder, and/or medication), then the International Classification of Sleep Disorders (third edition) published by the American Academy of Sleep Medicine classifies this condition as "hypersomnia due to a medical disorder" ${ }^{40}$ If posttraumatic hypersomnia is associated with two or more sleep onset rapid eye movement periods on multiple sleep latency testing, the diagnosis is "narcolepsy type 1 or type 2 due to a medical condition".

Other sleep disorders are also seen in patients who have suffered a head injury and complain of hypersomnia. Masel et al reviewed a total of 71 head injury patients in a residential treatment program, all without a prior history of sleep disturbances or hypersomnia. ${ }^{41}$ Among the 33 (46.5\%) hypersomnolent patients, four had obstructive sleep apnea, seven had periodic limb movement disorder, and one had 
narcolepsy (in addition to periodic limb movement disorder). The remaining patients were given a diagnosis of "posttraumatic hypersomnia". Guilleminault et al studied 184 traumatic brain injury patients and found that the majority of patients had objective sleepiness and only $17 \%$ of the patients had a normal mean sleep latency (greater than 10 minutes) on the multiple sleep latency test. Sleep-disordered breathing (primarily obstructive sleep apnea) was present in 32\% of the patients and all 16 whiplash patients were diagnosed with sleep-disordered breathing. In addition, pain was a significant cause of nocturnal sleep disruption and daytime impairment. ${ }^{18}$ Castriotta et al prospectively followed 87 adults for at least 3 months after traumatic brain injury. All subjects underwent polysomnography and multiple sleep latency testing. Fortysix percent of the patients had abnormal studies. Obstructive sleep apnea was diagnosed in $23 \%$ of the patients, $11 \%$ were found to suffer from posttraumatic hypersomnia, $7 \%$ had periodic limb movements in sleep, and 6\% met the criteria for narcolepsy. ${ }^{42}$

Although rare in occurrence, traumatic brain injury has also been reported to trigger cases of Kleine-Levin syndrome, a disorder involving recurrent episodes of hypersomnia and often accompanied by cognitive or behavioral disturbances, hypersexuality, and/or compulsive eating. ${ }^{43}$

\section{Parasomnias, fatigue, and mood disorders}

Traumatic brain injury occasionally precipitates parasomnias, including sleepwalking, sleep terrors, and rapid eye movement sleep behavior disorder. ${ }^{44}$ At times, a combination of rapid eye movement and non-rapid eye movement parasomnias produces a "parasomnia overlap disorder". Fatigue is also associated with traumatic brain injury with adverse effects on quality of life. Among the 119 patients studied at least 1 year after suffering a traumatic brain injury, up to $53 \%$ reported fatigue. This was more frequently reported in women or those with symptoms of depression, pain, or sleep disturbance. ${ }^{45}$ In another study of patients who had suffered a moderate-to-severe traumatic brain injury, 16\%-32\% and $21 \%-34 \%$ (at 1 and 2 years, respectively) reported significant levels of fatigue. ${ }^{46}$

Mood disorders may also occur after traumatic brain injury. Patients with mild traumatic brain injury and sleep complaints were more likely to report feeling depressed at 10 days and 6 weeks after their injury. ${ }^{47}$ In a large study of military personnel, a positive screening for traumatic brain injury and sleep problems was found to be an early indicator of risk for developing posttraumatic stress disorder and/or depression. ${ }^{48} \mathrm{New}-$ onset anxiety after a traumatic brain injury was a significant predictor of sleep disturbance, though the cause-effect relationship is unclear. ${ }^{49}$

\section{Diagnosis}

The patient's history is a crucial aspect of the workup to 1) document the association of the trauma with the sleep disorder, 2) rule out a preexisting sleep disorder, and 3) assess the progression of symptoms after the head injury. Physical examination is also important (Figure 1). Polysomnography should be considered if hypersomnia and/or other symptoms of sleep apnea are present. Cases of posttraumatic hypersomnia have shown an increase in sleep duration with or without changes in other sleep measures. ${ }^{50}$ Patients with a posttraumatic insomnia have shown long sleep latencies, low sleep efficiency, a decrease in nightly sleep duration, and an increase in stage- 1 sleep. ${ }^{34,51}$ Patients with moderate-tosevere traumatic brain injury showed increased slow-wave sleep, reduced rapid eye movement sleep, and more frequent nocturnal awakenings compared to controls. ${ }^{52}$ Continuous polysomnography has confirmed an increase in total sleep per 24 hours in some patients with posttraumatic hypersomnia. ${ }^{50}$ From a historical perspective, it is of interest to note that polysomnography during the comatose period has prognostic value for the development of full recovery without posttraumatic sleep disturbance. Normal amounts of sleep spindles, K-complexes, and normal cycling between non-rapid eye movement and rapid eye movement sleep are favorable prognostic signs. ${ }^{5,53,54}$ More recently, Valente et al also demonstrated that organized sleep patterns, but not Glasgow Coma Scale scores, were predictive of better prognosis during the subacute stages of posttraumatic coma. ${ }^{55}$

If daytime sleepiness is a major complaint, a multiple sleep latency test following polysomnogram may aid in determining if a central hypersomnia is present. In posttraumatic hypersomnia with daytime sleepiness, sleep latency may be markedly shortened. ${ }^{50}$ On the other hand, patients with posttraumatic insomnia exhibit prolonged sleep latencies on multiple sleep latency testing, as well as during the overnight sleep studies. ${ }^{51}$ As already mentioned, the presence of sleep-disordered breathing and sleep-onset rapid eye movement periods does not eliminate the possibility that the head trauma was the precipitating factor in the development of the sleep disorder. Since a driving accident may also occur due to prior existing sleepiness, a thorough inquiry is needed to appropriately compensate victims of secondary sleep disorders, even in the presence of preexisting risk factors. The medical workup should include assessment of the activity of the individual prior to the accident. This assessment 


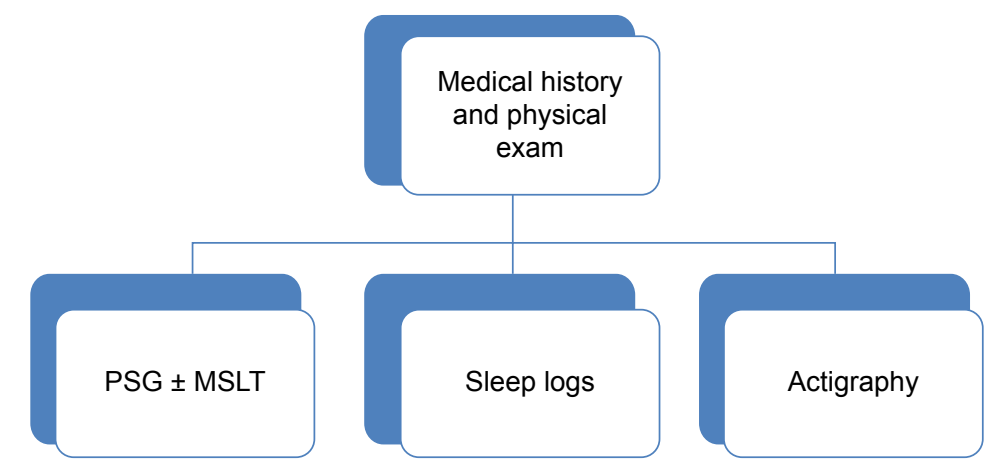

Figure I Diagnosis of sleep disturbances.

Abbreviations: PSG, polysomnogram; MSLT, multiple sleep latency test.

should include 1) interviews of bed partners and coworkers; 2 ) investigation of employer records and prior health and driving records; and 3) employment history, including missed work days. Head trauma is obviously a trigger of the syndrome in an individual with daytime sleepiness associated with sleep-disordered breathing who previously had an exemplary work record, full employment, and adequate driving records prior to the accident.

Obesity and craniofacial anatomical features can sometimes predispose a person to posttraumatic sleep disturbance and these should be noted on exam. ${ }^{18}$ Sleep diaries, in which a patient documents on a chart the times they attempted to sleep and how much sleep was attained, is a very useful tool. Finally, actigraphy, a device worn on the wrist to record movement, has been found to be a helpful adjunct to a sleep diary in those who have suffered a brain injury. ${ }^{56}$

\section{Treatment}

Posttraumatic insomnia may be challenging to treat (Figure 2). Benzodiazepines should be avoided secondary to their cognitive side effects, which may be compounded in a patient with traumatic brain injury. ${ }^{57}$ Furthermore, clinicians are often hesitant to prescribe benzodiazepines due to their risk of dependency and/or abuse. The non-benzodiazepine receptor agonists are extensively used, including zolpidem, the longer-acting eszopiclone, and the shorter-acting zaleplon. Although these medications are often helpful with initiating and/or maintaining sleep, they are associated with some concerning side effects, including complex sleeprelated behaviors (ie, sleepwalking and sleep-related eating disorder). A randomized, double-blinded, crossover trial involving traumatic brain injury and stroke patients treated with lorazepam $(0.5-1 \mathrm{mg})$ and zopiclone $(3.75-7.5 \mathrm{mg}$, another non-benzodiazepine receptor agonist available outside of the US) at bedtime found that these agents were equally effective. There were no major differences in sleep characteristics or cognitive performance on the Mini Mental Status Exam. ${ }^{58}$ In addition, many sedating antidepressants are used for insomnia, especially when comorbid depression exists. These medications include trazodone, mirtazapine, and doxepin. Suvorexant $\left(\right.$ Belsomra $^{\circledR}$; Merck \& Co Inc, Kenilworth, NJ, USA) was recently approved for the treatment of insomnia in the US and has a unique mechanism of action, which involves blocking the binding of wake-promoting neuropeptides (orexin A and B) to receptors (OX1R and OX2R). This medication should be used with care as it can lead to impaired motor coordination, complex sleep-related behaviors, mood/behavioral/cognitive changes, sleep paralysis, and/or cataplexy-like symptoms. Furthermore, patients are frequently treated with medications such as gabapentin, quetiapine, or olanzapine due to a well-known side effect of sleepiness, but there is no evidence of efficacy for their use in posttraumatic insomnia. Over-the-counter medications, such as diphenhydramine, doxylamine, and hydroxyzine (all first-generation antihistamines), are often used to aid sleep. Melatonin and various herbal supplements are also frequently utilized, including valerian, gamma-aminobutyric, 5-L-5hydroxytryptophan, kava, and many others. Overall, there are few studies comparing the effectiveness of these various agents. In a randomized, double-blind, controlled crossover trial comparing amitriptyline $(25 \mathrm{mg})$ and melatonin $(5 \mathrm{mg})$ in a small group of traumatic brain injury patients with chronic sleep disturbances, no difference was found in sleep duration/ latency/quality or daytime alertness. ${ }^{59}$ When using any of these medications/supplements, the prescriber should caution the patient not to drive (or do any other activity that could be of danger to self or others) after taking the medication. The patient should not use these medications with alcohol and use restraint when taking these with other sedating medications. It is also important to guide the patient to first 

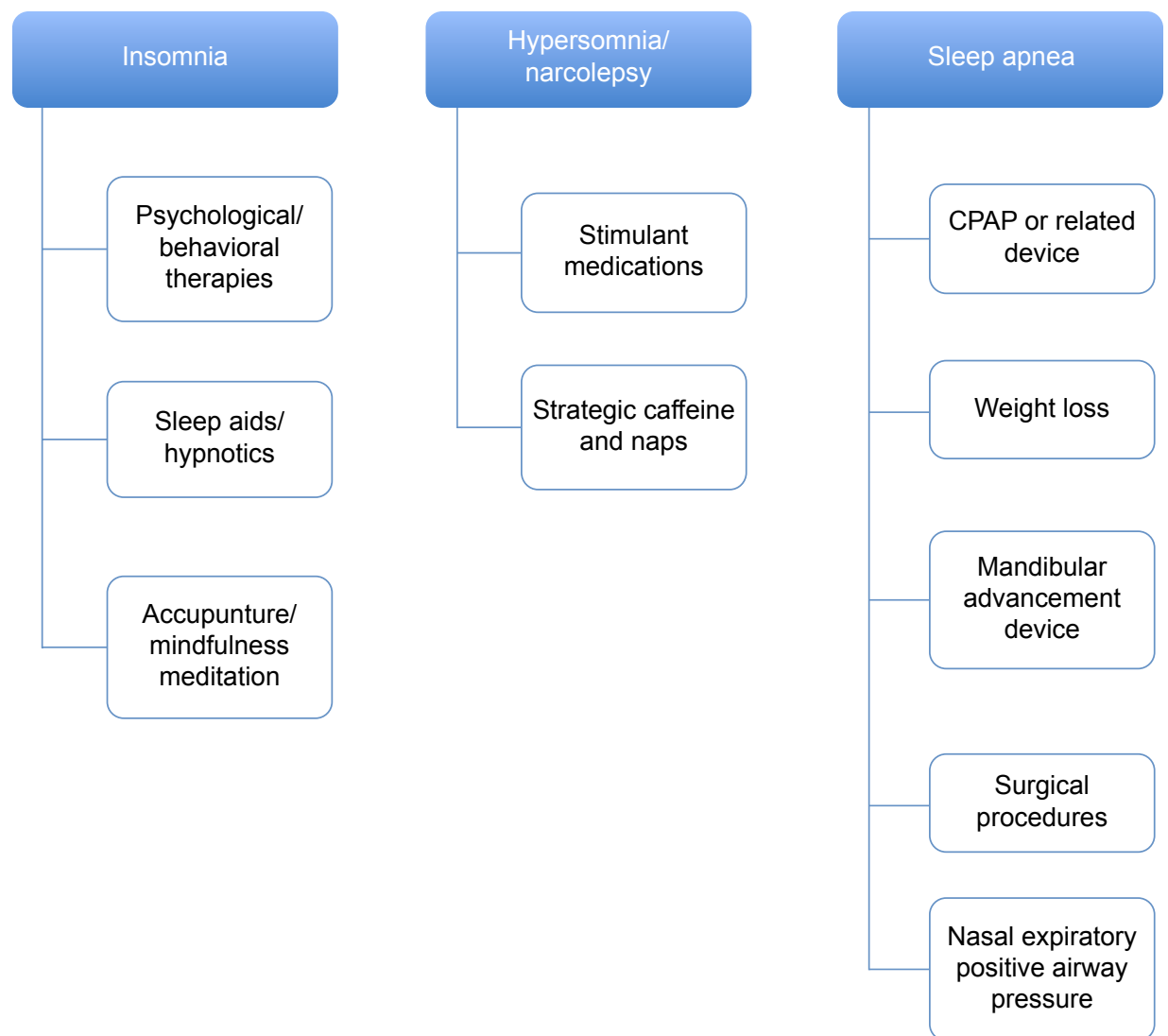

Figure 2 Treatment options for traumatic brain injury sleep disorders.

Notes: Adapted from Neurologic Clinics; 30(4); Viola-Saltzman M, Watson NF; Traumatic Brain Injury and Sleep Disorders; I299-1312; Copyright 20I2, with permission from Elsevier. ${ }^{75}$

Abbreviation: CPAP, continuous positive airway pressure.

try the medication on an evening when they do not need to be somewhere early the next day so that they can monitor for any residual sleepiness the following day.

In addition to pharmaceutical treatment, nonpharmaceutical techniques should be discussed and attempted for all insomnia patients. Psychological and behavioral therapies include, but are not limited to, stimulus control therapy, relaxation training, mindfulness meditation, and cognitive behavioral therapy. One study that examined 54 patients with chronic insomnia showed that a mindfulness meditation intervention significantly reduced total wake time as well as the insomnia severity index from baseline when compared to a self-monitoring control. ${ }^{60}$ Other modalities likely used with these are sleep restriction therapy, biofeedback, paradoxical intention, and sleep hygiene education. ${ }^{61}$ These therapies lead to improved nocturnal sleep quality and a reduction in daytime fatigue in those with insomnia related to traumatic brain injury. ${ }^{62}$ In addition, acupuncture is also a good treatment for improving sleep quality following traumatic brain injury. ${ }^{63}$

Patients with narcolepsy or hypersomnia due to a traumatic brain injury may benefit from the use of a stimulant medication. Modafinil may be the medication of first intention since it has fewer side effects than the other stimulants. Modafinil activates hypothalamic regions and is given in two divided doses (in the morning and around lunchtime). A prospective, double-blind, randomized, placebo-controlled trial found that modafinil (100-200 mg dose each morning) significantly improved sleepiness as measured by the Epworth Sleepiness Scale and the Maintenance of Wakefulness Test, although modafinil did not prove to be effective for fatigue. ${ }^{64}$ However, another study showed that modafinil had limited effectiveness for sleepiness associated with traumatic brain injury. ${ }^{65}$ Armodafinil (Nuvigi ${ }^{\circledR}$; Teva Pharmaceuticals, Petha Tikva, Israel), the R-enantiomer of modafinil, is dosed only once per day. Methylphenidate and amphetamines are also used in those with narcolepsy (or hypersomnia) related to a head injury. Patients with severe head trauma who complain of intellectual slowness may benefit more from amphetaminebased medications since these have a general "activating" effect that is not solely devoted to sleepiness. When cataplexy is present in narcolepsy, sodium oxybate may be prescribed since it is not only effective in the treatment of sleepiness, but it also consolidates sleep and may reduce the frequency of cataplexy/hypnagogic hallucinations/sleep paralysis. 
If this is not used, then a tricyclic antidepressant, selective serotonin reuptake inhibitor, or venlafaxine is prescribed to reduce cataplexy, if present. ${ }^{66}$ Undoubtedly, patients should be educated regarding strategic napping and caffeine use and not to drive if drowsy. Another non-pharmaceutical therapy may include daily blue-light therapy that was found to improve posttraumatic fatigue. ${ }^{67}$

Sleep apneas resulting from a traumatic brain injury are treated in the usual manner, usually with continuous positive airway pressure therapy. Sometimes the spontaneous/timed mode of bilevel positive airway pressure therapy or adaptive servoventilation is necessary for central sleep apnea or when both obstructive and central sleep apneas are present. Other treatments for obstructive sleep apnea may include mandibular advancement device (or oral appliance). These are used when mild or moderate obstructive sleep apnea is present and have a success rate of $\sim 50 \%$. They are less effective for severe obstructive sleep apnea and in those who are obese. Nasal expiratory positive airway pressure, a device applied to the nostrils during sleep, has also been proven effective to treat obstructive sleep apnea. Surgical procedures are considered at times, especially in those with severe obstructive sleep apnea or in those who have difficulty tolerating positive airway pressure. Surgical procedures include tonsillectomy, septoplasty, turbinate reduction, uvulopalatopharyngoplasty, genioglossus advancement and hyoid myotomy, and/or maxillomanibular advancement. Tracheostomy is rarely used for obstructive sleep apnea, and is usually reserved for life-threatening cases. Weight reduction surgeries are another surgical consideration in morbidly obese patients with obstructive sleep apnea. In addition, a newly approved device Inspire ${ }^{\circledR}$ (Inspire Medical Systems Inc, Minneapolis, MN, USA), an upper airway stimulation system, is used to treat obstructive sleep apnea. Finally, conservative treatments for obstructive sleep apnea include weight loss, avoiding supine position during sleep, and maintaining nasal patency with the use of saline spray or prescribed nasal steroid spray, if needed. One should also avoid alcohol and medications that may reduce muscle tone or influence the respiratory drive (eg, narcotics and benzodiazepines) prior to bedtime. ${ }^{68}$

Periodic limb movement disorder has been associated with traumatic brain injury and is treated in the same manner as restless legs syndrome. The dopamine agonists (ie, ropinirole or pramipexole) are commonly used to treat this disorder. Gabapentin, pregabalin (Lyrica ${ }^{\circledR}$; Pfizer Inc, New York City, NY, USA), and gabapentin encarbil (Horizant ${ }^{\mathbb{}}$; XenoPort Inc, Santa Clara, CA, USA) are also often used and may have a more tolerable side effect profile. Levodopa has been proven effective but due to the risk of augmentation, it is utilized less frequently. Narcotics and benzodiazepines are also effective, but are reserved for more significant cases that do not respond to other medications. Notably, iron storage levels should be checked in these patients to ensure a ferritin level of $50 \mathrm{ng} / \mathrm{mL}$ or greater. Iron supplementation is the treatment of choice if ferritin is less than desired. ${ }^{69}$

When treating a patient with a traumatic brain injury sleep disorder, the clinician must also address underlying pain, depression, and anxiety as these issues also impact sleep (Figure 3). Avoidance (or minimization) of narcotics and benzodiazepines is important as these medications can worsen traumatic brain injury-related sleep apnea. "Selfmedicating" with alcohol may lead to sleep disruption, nightmares, reduction in rapid eye movement sleep, and worsening sleep apnea. Selective serotonin reuptake inhibitors prescribed for depression or anxiety should be taken in the morning since they can induce insomnia when taken at bedtime. Finally, tricyclic antidepressants are often given to treat chronic pain issues and may also be helpful with insomnia due to their sedating effects.

\section{Prognosis}

There are few careful follow-up studies of patients with posttraumatic sleep disorders. The general clinical impression is that once stabilized, sleep disturbance related to organic brain damage shows little further change, other than a reversible response to treatment. A prospective cohort study examined sleepiness in 514 traumatic brain injury patients 1 month after the injury and then at 1 year. Sleepiness questions were extracted from sickness impact profile (SIP), a health-related quality-of-life questionnaire, which included the following: 1) I am sleeping or dozing most of the time - day or night; 2) I sit around half-asleep; 3) I sleep or nap more during the

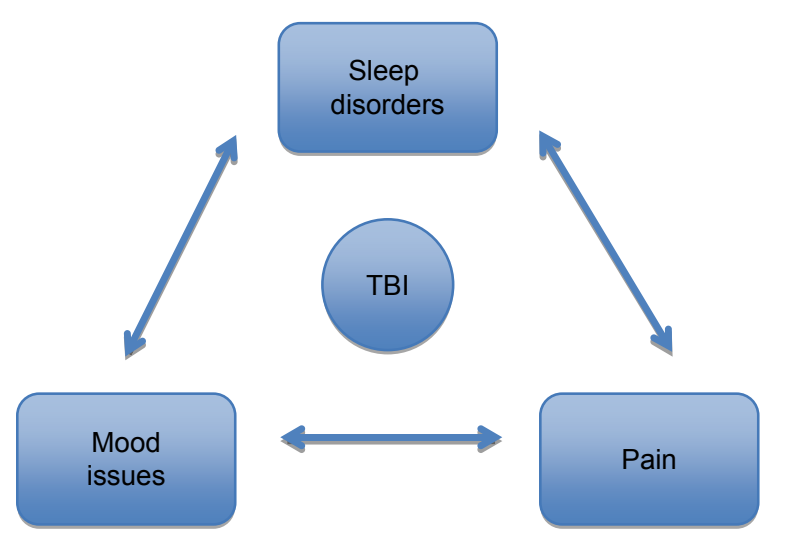

Figure 3 Relationship among sleep-related issues.

Notes: Adapted from Neurologic Clinics; 30(4); Viola-Saltzman M, Watson NF; Traumatic Brain Injury and Sleep Disorders; 1299-1312; Copyright 2012, with permission from Elsevier. ${ }^{75}$

Abbreviation: $\mathrm{TBI}$, traumatic brain injury. 
day; and 4) I sleep longer during the night. At 1 month, 55\% admitted to at least one item on this four-item sleepiness questionnaire. At 1 year, only $27 \%$ endorsed one or more of these sleepiness items. ${ }^{2}$ Another prospective study of 51 patients with traumatic brain injury demonstrated that at 3 -years postinjury, $67 \%$ of the patients continued to complain of sleep-wake disturbances, especially hypersomnia and fatigue. ${ }^{70}$ Disturbed nocturnal sleep may be a marker of more severe injury in patients with closed head injury. ${ }^{71}$ Improved sleep efficiency correlated with resolution of posttraumatic amnesia. ${ }^{72}$

In those with traumatic brain injury, the presence of obstructive sleep apnea was associated with more impairment of sustained attention and memory compared to patients with similar severity traumatic brain injury without obstructive sleep apnea. ${ }^{73}$ Traumatic brain injury patients with daytime hypersomnia had slower reaction times and poorer performance on the Psychomotor Vigilance Test than nonsleepy patients. $^{42}$

Unfortunately, treatment of sleep disorders associated with traumatic brain injury usually does not improve sleepiness or neuropsychological function. In an unselected group of 57 patients with traumatic brain injury, Castriotta et al documented sleep disorders in 22 subjects (39\%) using polysomnogram. Treatment did not lead to significant changes in quality of life, mood, or cognitive performance. Treatment of obstructive sleep apnea (13 subjects, 23\%) with continuous positive airway pressure did not lead to improvement in sleepiness, as measured by the Epworth Sleepiness Scale and multiple sleep latency test. ${ }^{74}$

\section{Conclusion}

Sleep disorders are prevalent in patients following traumatic brain injury. These symptoms and disorders include hypersomnia, insomnia, circadian rhythm disruption, parasomnias, fatigue, and mood alterations, which can also affect sleep. A careful history delineating head trauma prior to onset of sleep disruption as well as diagnostic tests such as polysomnogram, Multiple Sleep Latency Test, and/or actigraphy are important elements of making a diagnosis. Treatment is disorder specific; however, treatment of a sleep disorder such as obstructive sleep apnea may not lead to improvement in sleepiness or neurological dysfunction. This can make treatment of posttraumatic sleep disruption and its symptoms difficult and complex. Further studies are needed to help establish guidelines for the treatment of sleep disorders secondary to traumatic brain injuries.

\section{Disclosure}

The authors report no conflicts of interest in this work.

\section{References}

1. Ouellet MC, Savard J, Morin CM. Insomnia following traumatic brain injury: a review. Neurorehabil Neural Repair. 2004;18(4):187-198.

2. Watson NF, Dikmen S, Machamer J, Doherty M, Temkin N. Hypersomnia following traumatic brain injury. J Clin Sleep Med. 2007; 3(4):363-378.

3. Cohen M, Oksenberg A, Snir D, Stern MJ, Groswasser Z. Temporally related changes of sleep complaints in traumatic brain injured patients. J Neurol Neurosurg Psychiatry. 1992;55:313-315.

4. Winston SR. Preliminary communication: EMT and the Glasgow [correction of Glascow] Coma Scale. J Iowa Med Soc. 1979;69(10): 393-398.

5. Bricolo A, Gentilomo A, Rosadini G, Rossi GF. Long-lasting post-traumatic unconsciousness. A study based on nocturnal EEG and polygraphic recording. Acta Neurol Scand. 1968;44(4): $513-532$.

6. Langlois JA, Rutland-Brown W, Wald MM. The epidemiology and impact of traumatic brain injury: a brief overview. J Head Trauma Rehabil. 2006;21(5):375-378.

7. Schultz BA, Cifu DX, McNamee S, Nichols M, Carne W. Assessment and treatment of common persistent sequelae following blast induced mild traumatic brain injury. NeuroRehabilitation. 2011;28(4):309-320.

8. Baumann CR, Werth E, Stocker R, Ludwig S, Bassetti CL. Sleep-wake disturbances 6 months after traumatic brain injury: a prospective study. Brain. 2007;130(Pt 7):1873-1883.

9. Verma A, Anand V, Verma NP. Sleep disorders in chronic traumatic brain injury. J Clin Sleep Med. 2007;3(4):357-362.

10. Blinman TA, Houseknecht E, Snyder C, Wiebe DJ, Nance ML. Postconcussive symptoms in hospitalized pediatric patients after mild traumatic brain injury. J Pediatr Surg. 2009;44(6):1223-1228.

11. Hooper SR, Alexander J, Moore D, et al. Caregiver reports of common symptoms in children following a traumatic brain injury. NeuroRehabilitation. 2004;19(3):175-189.

12. Tham SW, Palermo TM, Vavilala MS, et al. The longitudinal course, risk factors, and impact of sleep disturbances in children with traumatic brain injury. J Neurotrauma. 2012;29(1):154-161.

13. Hou L, Han X, Sheng P, et al. Risk factors associated with sleep disturbances following traumatic brain injury: clinical findings and questionnaire based study. PLoS One. 2013;8(10):e76087.

14. Andriessen T, Jacobs B, Vos P. Clinical characteristics and pathophysiological mechanism of focal and diffuse traumatic brain injury. $J$ Cell Mol Med. 2010;14(10):2381-2392.

15. Lindsley DB, Bowden J, Magoun HW. Effect upon the EEG of acute injury to the brain stem activating system. Electroencephalogr Clin Neurophysiol. 1949;1:475-486.

16. Parizel PM, Ozsarlak, Van Goethem JW, et al. Imaging findings in diffuse axonal injury after closed head trauma. Eur Radiol. 1998;8(6): 960-965.

17. Hall CS, Danoff D. Sleep attacks: apparent relationship to atlantoaxial dislocation. Arch Neurol. 1975;32:58-59.

18. Guilleminault C, Yuen KM, Gulevich MG, Karadeniz D, Leger D, Philip P. Hypersomnia after head-neck trauma: a medicolegal dilemma Neurology. 2000;54:653-659.

19. Courville CB. Coup-contrecoup mechanism of cranio-cerebral injuries: some observations. Arch Surg. 1942;55:19-43.

20. Ommaya AK, Grubb RL, Naumann RA. Coup and contre-coup injury: observations on the mechanics of visible brain injuries in the rhesus monkey. J Neurosurg. 1971;35:503-516.

21. Sterman MB, Clemente CD. Forebrain inhibitory mechanisms: cortical synchronization induced by basal forebrain stimulation. Exp Neurol. 1962;6:91-102. 
22. Yaeger K, Alhilali L, Fakhran S. Evaluation of tentorial length and angle in sleep-wake disturbances after mild traumatic brain injury. AJR Am J Roentgenol. 2014;202:614-618.

23. Billiard M, Negri C, Baldy-Moulignier M, Roquefeuille B, Passouant $P$. Organisation du sommeil chz les sujets atteints d'inconscience posttraumatique chronique [Sleep organization in subjects with chronic post-traumatic unconsciousness]. Rev Electroencephalogr Neurophysiol Clin. 1979;9:149-152. French.

24. Carter KA, Lettieri CJ, Pena JM. An unusual cause of insomnia following IED-induced traumatic brain injury. J Clin Sleep Med. 2010;6(2):205-206.

25. Shekleton JA, Parcell DL, Redman JR, Phipps-Nelson J, Ponsford JL, Rajaratnam SM. Sleep disturbance and melatonin levels following traumatic brain injury. Neurology. 2010;74(21):1732-1738.

26. Llompart-Pou JA, Pérez G, Raurich JM, et al. Loss of cortisol circadian rhythm in patients with traumatic brain injury: a microdialysis evaluation. Neurocrit Care. 2010;13(2):211-216.

27. Dauvilliers Y, Baumann CR, Carlander B, et al. CSF hypocretin-1 levels in narcolepsy, Kleine-Levin syndrome, and other hypersomnias and neurological conditions. J Neurol Neurosurg Psychiatry. 2003; 74(12):1667-1673.

28. Nishino S, Kanbayashi T. Symptomatic narcolepsy, cataplexy and hypersomnia, and their implications in the hypothalamic hypocretin/ orexin system. Sleep Med Rev. 2005;9(4):269-310.

29. Rodrigues RN, Silva AA. Sonolência diurna excessiva pós-traumatismo de crânio: associação com movimentos periódicos de pernas e distúrbio de comportamento do sono REM: relato de caso. [Excessive daytime sleepiness after traumatic brain injury: association with periodic limb movements and REM behavior disorder: case report]. Arq Neuropsiquiatr. 2002;60:656-660. Portuguese.

30. Pillar G, Averbooch E, Katz N, Peled N, Kaufman Y, Shahar E. Prevalence and risk of sleep disturbances in adolescents after minor head injury. Pediatr Neurol. 2003;29(2):131-135.

31. Mahmood O, Rapport LJ, Hanks RA, Fichtenberg NL. Neuropsychological performance and sleep disturbance following traumatic brain injury. J Head Trauma Rehabil. 2004;19(5):378-390.

32. Lavigne G, Khoury S, Chauny JM, Desautels A. Pain and sleep in postconcussion/mild traumatic brain injury. Pain. 2015;156:S75-S85.

33. Williams BR, Lazic SE, Ogilvie RD. Polysomnographic and quantitative EEG analysis of subjects with long-term insomnia complaints associated with mild traumatic brain injury. Clin Neurophysiol. 2008;119: 429-438.

34. Ouellet MC, Morin CM. Subjective and objective measures of insomnia in the context of traumatic brain injury: a preliminary study. Sleep Med. 2006;7(6):486-497.

35. Ayalon L, Borodkin K, Dishon L, Kanety H, Dagan Y. Circadian rhythm sleep disorders following mild traumatic brain injury. Neurology. 2007; 68(14):1136-1140.

36. Bryan CJ. Repetitive traumatic brain injury (or concussion) increases severity of sleep disturbance among deployed military personnel. Sleep. 2013;36(6):941-946.

37. Ruff RL, Ruff SS, Wang XF. Improving sleep: initial headache treatment in OIF/OEF veterans with blast-induced mild traumatic brain injury. J Rehabil Res Dev. 2009;46(9):1071-1084.

38. Wallace DM, Shafazand S, Ramos AR, Carvalho DZ, Gardener H. Insomnia characteristics and clinical correlates in Operation Enduring Freedom/Operation Iraqi Freedom veterans with post-traumatic stress disorder and mild traumatic brain injury: an exploratory study. Sleep Med. 2011;12(9):850-859.

39. Ruff RL, Riechers RG, Wang XF, Piero T, Ruff SS. For veterans with mild traumatic brain injury, improved posttraumatic stress disorder severity and sleep correlated with symptomatic improvement. J Rehabil Res Dev. 2012;49(9):1305-1320.

40. American Academy of Sleep Medicine. International Classification of Sleep Disorders. 3rd ed. Darien, IL: American Academy of Sleep Medicine; 2014.
41. Masel BE, Scheibel RS, Kimbark T, Kuna ST. Excessive daytime sleepiness in adults with brain injuries. Arch Phys Med Rehabil. 2001;82(11): $1526-1532$

42. Castriotta RJ, Wilde MC, Lai JM, Atanasov S, Masel BE, Kuna ST. Prevalence and consequences of sleep disorders in traumatic brain injury. J Clin Sleep Med. 2007;3(4):349-356.

43. Arnulf I, Zeitzer JM, File J, Farber N, Mignot E. Kleine-Levin syndrome: a systematic review of 186 cases in the literature. Brain. 2005;128:2763-2776.

44. Schenck CH, Boyd JL, Mahowald MW. A parasomnia overlap disorder involving sleepwalking, sleep terrors, and REM sleep behavior disorder in 33 polysomnographically confirmed cases. Sleep. 1997;20(11): 972-981.

45. Englander J, Bushnik T, Oggins J, Katznelson L. Fatigue after traumatic brain injury: association with neuroendocrine, sleep, depression and other factors. Brain Inj. 2010;24:1379-1388.

46. Bushnik T, Englander J, Wright J. Patterns of fatigue and its correlates over the first 2 years after traumatic brain injury. J Head Trauma Rehabil. 2008;23(1):25-32.

47. Chaput G, Giguère JF, Chauny JM, Denis R, Lavigne G. Relationship among subjective sleep complaints, headaches, and mood alterations following a mild traumatic brain injury. Sleep Med.2009;10: 713-716.

48. Macera CA, Aralis HK, Rauh MJ, MacGregor AJ. Do sleep problems mediate the relationship between traumatic brain injury and the development of mental health symptoms after deployment? Sleep. 2013;36(1):83-90.

49. Rao V, Spiro J, Vaishnavi S, et al. Prevalence and types of sleep disturbances acutely after traumatic brain injury. Brain Inj. 2008;22(5): 381-386.

50. Guilleminault C, Faull KF, Miles L, van den Hoed J. Posttraumatic excessive daytime sleepiness: a review of 20 patients. Neurology. 1983; 33:1584-1589.

51. Manseau C, Broughton RJ. Severe head injury: long term effects on sleep, sleepiness and performance. Sleep Res. 1990;19:335.

52. Parcell DL, Ponsford JL, Redman JR, Rajaratnam SM. Poor sleep quality and changes in objectively recorded sleep after traumatic brain injury: a preliminary study. Arch Phys Med Rehabil. 2008;89(5): 843-850.

53. Bergamasco B, Bergamini L, Doriguzzi T. Clinical value of the sleep electroencephalographic patterns in posttraumatic coma. Acta Neurol Scand. 1968;44:495-511.

54. Lessard CS, Sances A, Larson SJ. Period analysis of EEG signals during sleep and posttraumatic coma. Aerospace Med. 1974;45:664-668.

55. Valente M, Placidi F, Oliveira AJ, et al. Sleep organization pattern as a prognostic marker at the subacute state of post-traumatic coma. Clin Neurophysiol. 2002;113:1798-1805.

56. Sinclair KL, Ponsford J, Rajaratnam SM. Actigraphic assessment of sleep disturbances following traumatic brain injury. Behav Sleep Med. 2014;12(1):13-27.

57. Buffett-Jerrott SE, Stewart SH. Cognitive and sedative effects of benzodiazepine use. Curr Pharm Des. 2002;8:45-58.

58. Li Pi Shan RS, Ashworth NL. Comparison of lorazepam and zopiclone for insomnia in patients with stroke and brain injury: A randomized, crossover, double-blinded trial. Am J Phys Med Rehabil. 2004; 83(6):421-427.

59. Kemp S, Biswas R, Neumann V, Coughlan A. The value of melatonin for sleep disorders occurring post-head injury: a pilot RCT. Brain Inj. 2004;18(9):911-919.

60. Ong JC, Manber R, Segal Z, Xia Y, Shapiro S, Wyatt JK. A randomized controlled trial of mindfulness meditation for chronic insomnia. Sleep. 2014;37(9):1553-1563.

61. Morgenthaler TI, Kramer M, Alessi C, et al. Practice parameters for the psychological and behavioral treatment of insomnia: an update. An American Academy of Sleep Medicine Report. Sleep. 2006;29(11): $1415-1419$ 
62. Ouellet MC, Morin CM. Efficacy of cognitive-behavioral therapy for insomnia associated with traumatic brain injury: a single-case experimental design. Arch Phys Med Rehabil. 2007;88(12):1581-1592.

63. Zollman FS, Larson EB, Wasek-Throm LK, Cyborski CM, Bode RK. Acupuncture for treatment of insomnia in patients with traumatic brain injury: a pilot intervention study. J Head Trauma Rehabil. 2012;27(2): 135-142.

64. Kaiser PR, Valko PO, Werth E, et al. Modafinil ameliorates excessive daytime sleepiness after traumatic brain injury. Neurology. 2010;75: 1780-1785.

65. Jha A, Weintraub A, Allshouse A, et al. A randomized trial of modafinil for the treatment of fatigue and excessive daytime sleepiness in individuals with chronic traumatic brain injury. J Head Trauma Rehabil. 2008;23(1):52-63.

66. Morgenthaler TI, Kapur VK, Brown TM, et al. Standards of Practice Committee of the American Academy of Sleep Medicine. Practice parameters for the treatment of narcolepsy and other hypersomnias of central origin. Sleep. 2007;30(12):1705-1711.

67. Sinclair KL, Ponsford JL, Taffe J, Lockley SW, Rajaratnam SMW. Randomized controlled trial of light therapy for fatigue following traumatic brain injury. Neurorehabil Neural Repair. 2014;28:303-313.

68. Morgenthaler TI, Kapen S, Lee-Chiong T, et al. Practice parameters for the medical therapy of obstructive sleep apnea. Sleep. 2006;29(8): 1031-1035.
69. Aurora RN, Kristo DA, Bista SR, et al. The treatment of restless legs syndrome and periodic limb movement disorder in adults - an update for 2012: practice parameters with an evidence-based systematic review and meta-analyses: an American Academy of Sleep Medicine Clinical Practice Guideline. Sleep. 2012;35(8):1039-1062.

70. Kempf J, Werth E, Kaiser PR, Bassetti CL, Baumann CR. Sleep-wake disturbances 3 years after traumatic brain injury. J Neurol Neurosurg Psychiatry. 2010;81:1402-1405.

71. Makley MJ, English JB, Drubach DA, Kreuz AJ, Celnik PA, Tarwater PM. Prevalence of sleep disturbance in closed head injury patients in a rehabilitation unit. Neurorehabil Neural Repair. 2008; 22(4):341-347.

72. Makley MJ, Johnson-Greene L, Tarwater PM, et al. Return of memory and sleep efficiency following moderate to severe closed head injury. Neurorehabil Neural Repair. 2009;23(4):320-326.

73. Wilde MC, Castriotta RJ, Lai JM, Atanasov S, Masel BE, Kuna ST. Cognitive impairment in patients with traumatic brain injury and obstructive sleep apnea. Arch Phys Med Rehabil. 2007;88(10):1284-1288.

74. Castriotta RJ, Atanasov S, Wilde MC, Masel BE, Lai JM, Kuna ST. Treatment of sleep disorders after traumatic brain injury. J Clin Sleep Med. 2009;5(2):137-144.

75. Viola-Saltzman M, Watson NF. Traumatic brain injury and sleep disorders. Neurol Clin. 2012;30(4):1299-1312.
Neuropsychiatric Disease and Treatment

\section{Publish your work in this journal}

Neuropsychiatric Disease and Treatment is an international, peerreviewed journal of clinical therapeutics and pharmacology focusing on concise rapid reporting of clinical or pre-clinical studies on a range of neuropsychiatric and neurological disorders. This journal is indexed on PubMed Central, the 'PsycINFO' database and CAS,

\section{Dovepress}

and is the official journal of The International Neuropsychiatric Association (INA). The manuscript management system is completely online and includes a very quick and fair peer-review system, which is all easy to use. Visit http://www.dovepress.com/testimonials.php to read real quotes from published authors. 